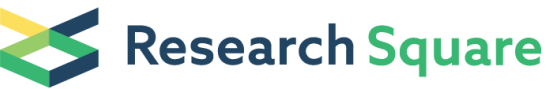 \\ Preprints are preliminary reports that have not undergone peer review. \\ They should not be considered conclusive, used to inform clinical practice, or referenced by the media as validated information.
}

\section{Diagnostic Accuracy Of Bedside Lung Ultrasound And Chest Radiography Compared To Thoracic Computed Tomography In Critically III Patients}

\section{Fernando Ariel Sosa}

Hospital Alemán: Hospital Aleman

Mercedes Kleinert

Hospital Alemán: Hospital Aleman

\section{Agustín Matarrese}

Hospital Alemán: Hospital Aleman

\section{Juan Criniti}

Hospital Alemán: Hospital Aleman

Juan José Folco

Hospital Alemán: Hospital Aleman

\section{Santiago Saavedra}

Hospital Alemán: Hospital Aleman

\section{Graciela Dorotesky}

Hospital Alemán: Hospital Aleman

\section{Javier Roberti ( $\nabla$ javierroberti@gmail.com )}

CONICIT: Consejo Nacional de Investigaciones Cientificas y Tecnologicas https://orcid.org/0000-0002-42855061

\section{Francisco Tamagnone}

Hospital Alemán: Hospital Aleman

\section{Research Article}

Keywords: lung ultrasound, chest radiography, tomography, intensive care, consolidation syndrome, interstitial syndrome, pleural effusion, pneumothorax.

Posted Date: February 28th, 2022

DOI: https://doi.org/10.21203/rs.3.rs-1125288/v1

License: (c) (P) This work is licensed under a Creative Commons Attribution 4.0 International License. Read Full License 


\section{Abstract}

Background: Bedside lung ultrasound (LUS) is being adopted in intensive care units (ICU) with a higher diagnostic accuracy for consolidation, interstitial syndrome, pleural effusion, and pneumothorax than chest radiography (CXR).

Aims: To compare the diagnostic performance of LUS and CXR for the detection of pathologic abnormalities in critically ill patients in the ICU using thoracic computed tomography (CT) as the gold standard.

Methods. Prospective and descriptive single-center study. Prior to CT, we performed bedside LUS and CXR, evaluated hemithoraces and characterized them as positive or negative for each of four abnormalities (consolidation syndrome, interstitial syndrome, pleural effusion, and pneumothorax). We decided therapeutic intervention after CT.

Results. A total of 58 patients and 126 hemithoraces were evaluated by three imaging techniques. LUS and CT did not show significant differences in any of the conditions. CRX and CT showed significantly different proportions of consolidation, interstitial syndrome and pleural effusion. LUS performed better than CXR, with a higher sensitivity for four pathologies. CXR had a marginally higher specificity than LUS for consolidation, interstitial syndrome and pneumothorax. In $79 \%$ of patients, we performed a subsequent action based on the information provided by LUS.

Conclusion. In ICU patients, bedside LUS offers a better diagnostic performance than CRX for the diagnosis of common pathologic conditions and could be an alternative to CT.

\section{Introduction}

Lung imaging in critically ill patients is performed either by bedside chest radiography (CXR) or thoracic computed tomography (CT) [1]. All approaches have limitations that could lead to misdiagnosis and adverse outcomes [1-4]. CXR requires patients to be moved to a difficult position, while to perform a CT, which is the most sensitive method, the patient needs to be transported out of the intensive care unit (ICU). Bedside lung ultrasound (LUS) is being adopted in ICUs, with a higher diagnostic accuracy for consolidation, interstitial syndrome, pleural effusion, and pneumothorax than $\operatorname{CXR}[3,5,6]$. Although portable CXR lacks the sensitivity and specificity of CT, it is yet widely used in some settings because of its low cost and speed [7, 8]. LUS does not require the demanding positioning of $\mathrm{CXR}$, and unlike $\mathrm{CT}$, the equipment can be brought into the ICU. LUS compares to the accuracy of chest $\mathrm{CT}$ for respiratory findings and offers physicians a radiation-free tool [9-15].

International guidelines support the use of point-of-care LUS to diagnose pathological abnormalities in critically ill patients [16-19]. The reported sensitivity and specificity of LUS, when compared to CT, was high among hospitalized patients with acute respiratory failure, and its sensitivity was better than that of CXR. However, paired comparisons in individual patients are scarce, and published studies have methodological limitations, such as a high risk of selection bias [9]. Our aim was to compare the diagnostic performance of lung ultrasound (LUS) and bedside CXR for the detection of pathologic abnormalities in critically ill patients in the ICU, using thoracic CT as the gold standard, and describing the clinical decisions made after detecting new infections with this technique. 


\section{Method}

This was a prospective and descriptive single-center study carried out from November 2018 to November 2019 at a medical-surgical ICU of a teaching hospital in Buenos Aires. The ICU admits patients who require hemodynamic, respiratory, circulatory, renal assistance, or special observation support.

\section{Patients}

We included patients admitted to the ICU, over 18 years of age, with a requirement for mechanical ventilation (MV) for at least 12 hours. Patients were enrolled if a physician not involved in this study had prescribed a thoracic CT because of clinical deterioration translated as any of the following events: (i) a suspicion of respiratory infection because of fever $\geq 38^{\circ} \mathrm{C}$, hemodynamic deterioration, systolic blood pressure of $<90 \mathrm{mmHg}$ with new onset or increase of vasopressor drugs, alterations in gas exchange manifested by a decrease in $\mathrm{PaO}_{2} / \mathrm{FiO}_{2}$ or the need for an increase in $\mathrm{FiO}_{2}$, change in the characteristics and volume of respiratory secretions; (ii) suspected barotrauma (pneumothorax); (iii) suspicion of increased extravascular lung water; (iv) a suspicion of pleural effusion. Patients were excluded if they were under 18 years of age, did not require MRA, were on a ventilator for less than 12 hours or had a confirmed diagnosis of pneumothorax, pulmonary condensation, acute lung edema or pleural effusion on admission. Prior to CT, we performed bedside LUS and CXR, evaluated hemithoraces and characterized them as positive or negative for each of four abnormalities (consolidation syndrome, interstitial syndrome, pleural effusion, and pneumothorax). We decided therapeutic intervention after CT.

\section{Chest radiography}

We performed anterior CXR with portable X-ray equipment (Siemens polymobile, Erlangen, Germany). An expert radiologist blinded to the lung ultrasound and CT findings assessed CXRs. Consolidation, interstitial syndrome, pneumothorax, and pleural effusion were defined using the terminology of the Nomenclature Committee of the Fleischner Society. As per the protocol at our institution, we performed CRX daily on patients with mechanical ventilation assistance.

\section{Lung ultrasound}

For LUS, we used a microconvex 5-9 MHz transducer. Two expert operators, unaware of the CT and CXR findings, evaluated ultrasonography. Patients were studied in the supine position; the lateral position was used for posterior lung surface examination. The anterior surface of each lung was defined by the clavicle, parasternal, anterior axillary line, and diaphragm and was divided into upper and lower areas. The lateral surface was defined by the anterior and posterior axillary lines and divided into upper and lower areas. The posterior lung surface was defined by the posterior axillary and paravertebral lines and divided into upper and lower areas. We scanned the apex from the supraclavicular space. The normal lung generates lung sliding and A-lines (repetition lines parallel to the pleural line). Two operators, experts in critical ultrasonography, evaluated the results.

\section{Definitions}

\section{Consolidation}

Defined as the presence of atelectasis, alveolar consolidation, or parenchymal bands. Lung regions were located using the same anatomical landmarks as with CRX. We used power Doppler to differentiate tissue-like structures 
from consolidation.

\section{Pneumothorax}

Diagnosed when the A-line sign (only A-lines visible) was associated with the stratosphere sign (complete abolition of lung sliding). Local lung sliding or B-lines exclude this diagnosis. The lung point sign, specific for pneumothorax, was also used.

\section{Pleural effusion}

Determined as a hypoechoic or echoic structure containing isoechoic particles or septations in inflammatory pleural diseases. In addition to power Doppler, the quad and sinusoid signs, showing pleural effusion regardless of echogenicity, were used.

\section{Interstitial syndrome}

Defined as the presence of ground-glass opacities, septal or nonseptal lines, or fibrotic changes. Table 1. Definition of agreement among methods

Table 1

Definition of agreement among methods

\begin{tabular}{|lll|}
\hline CRX & LUS & CT \\
\hline Normal & A-lines + lung sliding & Normal \\
\hline Interstitial & B1, B2, or B3 profile & Interstitial \\
\hline Ground glass & B2, B3, Atl/Cons & Consolidation \\
\hline Effusion & Effusion & Effusion \\
\hline Absence interstitial pattern, hyper clarity. & Absence lung sliding, bar code sign m mode & Pneumothorax \\
\hline Abbreviations: CRX, chest radiography; LUS, lung ultrasound; CT, computed tomography & \\
\hline
\end{tabular}

\section{Multiple-detector computed tomography (MDCT)}

We performed MDCT with a Siemens Somatom Sensation (16 slices, Erlangen, Germany). Scans were obtained in the supine position from the apex of the thorax to the lung bases. Assessments included thin MDCT highresolution computed tomography (MDCT-HRCT) and spiral MDCT scans. MDCT-HRCT scans were used to reveal diffuse lung parenchymal involvement as ground-glass opacities, septal or nonseptal lines, and fibrotic changes (architectural distortion). We evaluated scans for mediastinal and pleural pathology and lung lesions (atelectasis, alveolar consolidation, and parenchymal bands) as described by the Nomenclature Committee of the Fleischner Society. Two individual radiologists, unaware of the lung ultrasound and CXR findings, studied the MDCT-HRCT and MDCT images together, and decisions were reached by consensus.

\section{Ethical aspects}

The study was evaluated by the Independent Ethics Committee of the German Hospital. We obtained informed consent from the patients or their representatives. The investigators of this study complied with Law 


\section{Statistical analysis}

Continuous variables are expressed as the mean and standard deviation; categorical variables are expressed as absolute frequencies and percentages. We compared continuous variables using Student's t-test or Wilcoxon rank-sum test, depending on their distribution. For categorical variables, the chi-square test or Fisher's exact test was used. ROC area, sensitivity, and specificity were calculated using standard formulas. We conducted univariate and multivariate analyses to identify factors associated with implementing a specific subsequent action. A p-value $<0.05$ was considered statistically significant. All analyses were performed in Stata v14 (College Station, TX, USA).

\section{Results}

A total of 58 patients, 126 hemithoraces ( 2 in each patient), were evaluated by three imaging techniques. Table 2 shows main characteristics of patients. Of the 58 patients, 22 (39\%) were women, and the mean age was $65.7 \pm 15.6$ years. The mean value of Apache II was $16.5 \pm 5.7$, and that of Charlson was $4.7 \pm 2.1$. The most frequent causes of hospitalization in the ICU were liver transplantation, with $4(7.1 \%)$ cases; upper gastrointestinal bleeding, with $3(5,4 \%)$; and abdominal surgery, with $3(5.4 \%)$. The median time from ICU admission to respiratory events was 3 days (range, 1-84 days).

Table 2

Patients' characteristics

\begin{tabular}{|ll|}
\hline Characteristic & Value \\
\hline Age (years) mean \pm SD & $65.7 \pm 15.6$ \\
\hline Sex (female/male) & $22: 34(39.3 \%)$ \\
\hline APACHE II, mean \pm SD & $16.5 \pm 5.7$ \\
\hline Charlson, mean \pm SD & $4.7 \pm 2.1$ \\
\hline Admission diagnosis & \\
\hline Postoperative, $\mathrm{n}(\%)$ & $14(25)$ \\
\hline Acute respiratory failure, $\mathrm{n}(\%)$ & $19(33)$ \\
\hline Other, $\mathrm{n}(\%)$ & $23(41)$ \\
\hline
\end{tabular}

Using CT, consolidation was present in 100 (89.3\%) of the evaluated hemithoraces, followed by pleural effusion in $74(66.1 \%)$, interstitial syndrome in $40(35.7 \%)$ and pneumothorax in $4(3.6 \%)$. When comparing proportions, LUS and CT did not show statistically significant differences in any of the conditions; however, the comparison of $\mathrm{CRX}$ and $\mathrm{CT}$ showed significantly different proportions of detected consolidation, interstitial syndrome and pleural effusion. Table 3 shows an analysis of the proportion of the four conditions according to each method. 
Table 3

Results of test of proportions of LUS and CRX compared to CT

\begin{tabular}{|llllll|}
\hline Diagnosis & $\begin{array}{l}\text { LUS, } \\
\text { proportion }\end{array}$ & $\begin{array}{l}\text { P value difference } \\
\text { with CT }\end{array}$ & $\begin{array}{l}\text { CRX, } \\
\text { proportion }\end{array}$ & $\begin{array}{l}\text { P value difference } \\
\text { with CT }\end{array}$ & $\begin{array}{l}\text { CT, } \\
\text { proportion }\end{array}$ \\
\hline Consolidation & 0.83 & 0.176 & 0.53 & 0.00 & 0.89 \\
\hline $\begin{array}{l}\text { Interstitial } \\
\text { syndrome }\end{array}$ & 0.42 & 0.340 & 0.24 & 0.05 & 0.36 \\
\hline Pleural effusion & 0.56 & 0.132 & 0.52 & 0.03 & 0.66 \\
\hline Pneumothorax & 0.04 & 0.730 & 0.03 & 0.72 & 0.004 \\
\hline Abbreviations: CT, computed tomography; CRX, chest radiography; LUS, lung ultrasound & \\
\hline
\end{tabular}

Table 3

Sensitivity and specificity analysis of LUS and CRX compared to TAC

\begin{tabular}{|c|c|c|c|c|c|c|c|c|c|}
\hline Abnormality & Technique & $\begin{array}{l}\text { ROC } \\
\text { Area }\end{array}$ & SE & $\begin{array}{l}95 \% \\
\mathrm{Cl}\end{array}$ & Sensitivity & Specificity & $\begin{array}{l}\text { Correctly } \\
\text { classified }\end{array}$ & LR+ & LR- \\
\hline \multirow[t]{2}{*}{ Consolidation } & LUS & 0.82 & 0.07 & $\begin{array}{l}0.74- \\
0.89\end{array}$ & $90.0 \%$ & $75 \%$ & $88.4 \%$ & 3.6 & 0.1 \\
\hline & CRX & 0.75 & 0.05 & $\begin{array}{l}0.66- \\
0.83\end{array}$ & $59.2 \%$ & $91.7 \%$ & $62.7 \%$ & 7.1 & 0.4 \\
\hline \multirow[t]{2}{*}{$\begin{array}{l}\text { Interstitial } \\
\text { syndrome }\end{array}$} & LUS & 0.85 & 0.03 & $\begin{array}{l}0.78- \\
0.92\end{array}$ & $87.5 \%$ & $83.3 \%$ & $84.8 \%$ & 5.3 & 0.2 \\
\hline & CRX & 0.69 & 0.04 & $\begin{array}{l}0.60- \\
0.78\end{array}$ & $47.5 \%$ & $90 \%$ & $74.6 \%$ & 4.8 & 0.6 \\
\hline \multirow[t]{2}{*}{$\begin{array}{l}\text { Pleural } \\
\text { effusion }\end{array}$} & LUS & 0.87 & 0.03 & $\begin{array}{l}0.79- \\
0.92\end{array}$ & $81.1 \%$ & $92.1 \%$ & $84.8 \%$ & 10.3 & 0.2 \\
\hline & CRX & 0.67 & 0.05 & $\begin{array}{l}0.58- \\
0.76\end{array}$ & $63.9 \%$ & $71.1 \%$ & $66.4 \%$ & 2.2 & 0.5 \\
\hline \multirow[t]{2}{*}{ Pneumothorax } & LUS & 0.99 & 0.00 & $\begin{array}{l}0.95- \\
0.99\end{array}$ & $100 \%$ & $99.1 \%$ & $99.1 \%$ & 108.0 & 0.0 \\
\hline & CRX & 0.88 & 0.13 & $\begin{array}{l}0.80- \\
0.93\end{array}$ & $75 \%$ & $100 \%$ & $99.1 \%$ & - & 0.3 \\
\hline
\end{tabular}

The sensitivity, specificity, and correctly classified percentages of the four pathologies by LUS, CRX and CXR are shown in Table 4. LUS performed better than CXR, showing a significantly higher sensitivity for all four pathologies. CXR had a marginally higher specificity than LUS for consolidation, interstitial syndrome and pneumothorax. 
Table 4

Univariate analysis for subsequent action

\begin{tabular}{|lll|}
\hline Variable & RR (95\% Cl) & P value \\
\hline Age $\geq 70$ yr. & $0.82(0.59-1.11)$ & 0.20 \\
\hline Male gender & $1.08(0.76-1.53)$ & 0.67 \\
\hline Apache score $\geq 18$ pt. & $1.49(1.08-2.05)$ & 0.01 \\
\hline CCI $\geq 4$ pt. & $1.02(0.70-1.49)$ & 0.92 \\
\hline Clinical admission & $0.82(0.59-1.13)$ & 0.30 \\
\hline Consolidation in LUS & $1.10(0.61-1.98)$ & 0.74 \\
\hline Consolidation in CT & $2.08(0.84-5.15)$ & 0.03 \\
\hline Agreement among LUS observers & $1.52(0.95-2.44)$ & 0.05 \\
\hline Agreement among CRX observers & $1.40(1.19-1.65)$ & 1.00 \\
\hline Agreement LUS - CT & $1.16(0.76-1.79)$ & 0.43 \\
\hline CCl=Charlson's Comorbidity Index & & \\
\hline
\end{tabular}

\section{Decision-making}

In 42 (72\%) patients, the information provided by imaging studies led to a specific action. The most frequent actions were the performance of blood culture tests and the empirical administration of antibiotics. Among those cases in which a specific action was taken, in 29 (69\%) cases, the decision was made based on a diagnosis only detected by LUS but later confirmed by CT. In four (10\%) patients, both LUS and CRX provided the right diagnosis. CRX was the only useful method in 2 (5\%) cases. In the remaining 7 (17\%) patients, only the information provided by the CT was relevant to prescribe treatment or perform a diagnostic procedure. Overall, in $33(79 \%)$ patients, we performed a subsequent action based on the information provided by LUS. LUS was concordant with CT in 11 (69\%) patients who did not require any specific action.

When exploring predictors for subsequent clinical actions, we observed that, in univariate analysis, an increased Apache score at admission and a consolidation observed in CT were associated with a subsequent action after image workup. Agreement between both LUS observers showed a clear trend but did not reach statistical significance (Table 4).

\section{Discussion}

We found that LUS had a high diagnostic accuracy in common pulmonary abnormalities in critically ill patients; indeed, LUS showed a higher percentage of correct diagnosis of consolidation, interstitial syndrome, and pleural effusion than when using CRX as a diagnostic tool and a similar percentage of correct diagnosis pneumothorax at $99.1 \%$ when compared to CRX. In our study, LUS had a sensitivity of $90 \%$ and an accuracy of $88.4 \%$ for the detection of consolidation, which was comparable to previous studies $[5,6,10]$. The same occurred with the LUS accuracy for the detection of interstitial syndrome and pleural effusion when compared with CRX; LUS was 
significantly better, as reported in the literature $[5,6,11]$. Indeed, CRX showed low sensitivity for these abnormalities, with $59 \%, 48 \%$, and $64 \%$ sensitivity for consolidation, interstitial syndrome and pleural effusion, respectively $[5,6,11]$. It has also been suggested that LUS was better than CRX in differentiating forms of pleural effusions on the basis of internal echogenicity, homogeneity, and pleural thickness [12]. With pneumothorax, however, LUS and CRX were both very accurate. This finding is in contrast with a previous comparable study, in which CRX could not identify this abnormality [6]. It has been shown that LUS outperforms CRX when detecting residual pneumothoraxes after drainage and that residents can learn to operate on LUS after only two hours of training $[13,14]$.

Replacing CXR with LUS in the ICU reduces radiation exposure, which is a substantial improvement in patient safety [15]. Indeed, the carcinogenic effects of X-rays are well known; one chest CT scan has an effective radiation dose equivalent to 400 CXRs [20]. indeed, medical radiation from CRX and nuclear medicine is the most important source of radiation exposure in Western countries [20]. In our study, we performed 610 CRXs, and some patients spent several days in the ICU with substantial radiation exposure. Routine daily CXRs for critically ill patients have been the standard practice in many institutions. A recent intervention to promote a change in CXR ordering practice contributed to a decrease in routine CXRs and a two-thirds decrease in patients receiving CRXs [8].

Other studies have shown that the use of LUS reduced the use of CRX [21] or CRX and CT [21, 22]. Brogi et al. evaluated the influence of routine use of LUS on costs and the usage of CXRs in the ICU and found a significant reduction in the number of CXRs and in costs without affecting the outcome [21]. Additionally, the point-of-care LUS protocol led to a significantly lower utilization of chest radiography, ultrasound performed by non-intensivist specialists, and CT scans, leading to lower radiation exposure, less intrahospital transportation of unstable patients, and cost savings [22].

We found LUS to be a useful method to inform intensivists on the decision to prescribe a diagnostic and/or therapeutic procedure. LUS proved to inform the decision correctly in the vast majority of patients. In $76 \%$ of patients, performing CT did not provide any additional information that would change the decision based on the LUS findings. Agreement between LUS and CT was high, and coinciding with the literature, LUS alone informed the decision-making process [23]. This saved time and provided a better use of healthcare resources by avoiding unnecessary CRXs and CTs. Similar to other studies, we also found LUS to be superior to CRX [24].

This study has limitations that need to be acknowledged. First, we studied a small number of patients, which led to some constraints in the analysis. The location of the pathological finding and the analysis to identify factors associated with a subsequent action were both affected by the sample size. Second, the time interval between LUS and CT was minimized but could not be controlled. Third, this is a single center study, which could limit the transferability of our findings to other settings, especially those related to decision-making.

In conclusion, in ICU patients, bedside LUS, which can be performed by ICU specialists, offers better diagnostic performance than radiography for the diagnosis of common pathologic conditions and could be an alternative to CT scans.

\section{Declarations}

\section{i. Funding No funding}


ii. Conflicts of interest/Competing interests The authors do not have any conflict of interests to declare in relation to this study.

iii. Ethics approval Ethics committee approved protocol and IC

iv. Consent to participate Patients or next of kin gave consent

v. Consent for publication Patients or next of kin gave consent

vi. Availability of data and material available upon request

vii. Code availability NA

viii. Authors' contributions:

Sosa and Kleinert: concept idea, investigation, data collection, data analysis, interpretation, reviewing. Matarrese: data analysis, presentation, reviewing. Criniti, data collection, data interpretation, reviewing. Folco, data collection, data interpretation, reviewing. Saavedra, data collection, data interpretation, reviewing. Dorotesky, data collection, data interpretation, reviewing.Roberti, Javier, data interpretation, writing, reviewing. Tamagnone, data collection, data interpretation, reviewing.

Declaration of interest: The authors declare they do not have any conflict of interest in relation to this study.

Acknowledgments: none

\section{References}

1. Rubinowitz AN, Siegel MD, Tocino I. Thoracic imaging in the ICU. Crit Care Clin. 2007;23(3):539-73.

2. Beards SC, Jackson A, Hunt L, Wood A, Frerk CM, Brear G, et al. Interobserver variation in the chest radiograph component of the lung injury score. Anaesthesia. 1995;50(11):928-32.

3. Lichtenstein DA, Meziere GA. Relevance of lung ultrasound in the diagnosis of acute respiratory failure: the BLUE protocol. Chest. 2008;134(1):117-25.

4. Gazon M, Eboumbou N, Robert MO, Branche P, Duperret S, Viale JP. [Agreement between lung ultrasonography and chest radiography in the intensive care unit]. Ann Fr Anesth Reanim. 2011;30(1):6-12.

5. Lichtenstein DA, Lascols N, Meziere G, Gepner A. Ultrasound diagnosis of alveolar consolidation in the critically ill. Intensive Care Med. 2004;30(2):276-81.

6. Xirouchaki N, Magkanas E, Vaporidi K, Kondili E, Plataki M, Patrianakos A, et al. Lung ultrasound in critically ill patients: comparison with bedside chest radiography. Intensive Care Med. 2011;37(9):1488-93.

7. Sharifpour A, Alaee A, Aliyali M, Abedi S, Karimi N. Comparison of the Diagnostic and Therapeutic Efficacies of Portable Recruited Chest Radiography with Conventional Portable Radiography in Mechanically Ventilated Patients. Tanaffos. 2019;18(4):351-4.

8. Wu Y, Rose MQ, Freeman ML, Richard-Lany NP, Spaulding AC, Booth SC, et al. Reducing chest radiography utilization in the medical intensive care unit. J Am Assoc Nurse Pract. 2020;32(5):390-9.

9. Hew M, Corcoran JP, Harriss EK, Rahman NM, Mallett S. The diagnostic accuracy of chest ultrasound for CTdetected radiographic consolidation in hospitalised adults with acute respiratory failure: a systematic 
review. BMJ Open. 2015;5(5):e007838.

10. Haggag YI, Mashhour K, Ahmed K, Samir N, Radwan W. Effectiveness of Lung Ultrasound in Comparison with Chest X-Ray in Diagnosis of Lung Consolidation. Open Access Maced J Med Sci. 2019;7(15):2457-61.

11. Remerand F, Dellamonica J, Mao Z, Ferrari F, Bouhemad B, Jianxin Y, et al. Multiplane ultrasound approach to quantify pleural effusion at the bedside. Intensive Care Med. 2010;36(4):656-64.

12. Brogi E, Gargani L, Bignami E, Barbariol F, Marra A, Forfori F, et al. Thoracic ultrasound for pleural effusion in the intensive care unit: a narrative review from diagnosis to treatment. Crit Care. 2017;21(1):325.

13. Galbois A, Ait-Oufella H, Baudel JL, Kofman T, Bottero J, Viennot S, et al. Pleural ultrasound compared with chest radiographic detection of pneumothorax resolution after drainage. Chest. 2010;138(3):648-55.

14. Volpicelli G. Sonographic diagnosis of pneumothorax. Intensive Care Med. 2011;37(2):224-32.

15. Bitar ZI, Maadarani OS, El-Shably AM, Al-Ajmi MJ. Diagnostic accuracy of chest ultrasound in patients with pneumonia in the intensive care unit: A single-hospital study. Health Sci Rep. 2019;2(1):e102.

16. Shrestha GS, Weeratunga D, Baker K. Point of Care Ultrasound in Critically III Patient. Rev Recent Clin Trials. 2018 Jan 31;13(1):15-26.

17. Daniel A. Lichtenstein, Nathalie Lascols, Gilbert Mezire, Agns Gepner. Ultrasound diagnosis of alveolar consolidation in the critically ill. Intensive Care Med (2004) 30:276-281.

18. Charlotte Arbelota, Fabio Ferraria,b, Belaı"d Bouhemada and Jean-Jacques Rouby. Lung ultrasound in acute respiratory distress syndrome and acute lung injury. Current Opinion in Critical Care 2008, 14:70-74.

19. Philipp Enghard, Sibylle Rademacher, Jens Nee, Dietrich Hasper, Ulrike Engert, Achim Jörresand Jan M Kruse. Simplified lung ultrasound protocol shows excellent prediction of extravascular lung water in ventilated intensive care patients. Critical Care 2015. 19:36.

20. Picano E. Sustainability of medical imaging. BMJ. 2004;328(7439):578-80.

21. Brogi E, Bignami E, Sidoti A, Shawar M, Gargani L, Vetrugno L, et al. Could the use of bedside lung ultrasound reduce the number of chest x-rays in the intensive care unit? Cardiovasc Ultrasound. 2017;15(1):23.

22. Pontet J, Yic C, Diaz-Gomez JL, Rodriguez P, Sviridenko I, Mendez D, et al. Impact of an ultrasound-driven diagnostic protocol at early intensive-care stay: a randomized-controlled trial. Ultrasound J. 2019;11(1):24.

23. Chiumello D, Umbrello M, Sferrazza Papa GF, Angileri A, Gurgitano M, Formenti P, Coppola S, Froio S, Cammaroto A, Carrafiello G. Global and Regional Diagnostic Accuracy of Lung Ultrasound Compared to CT in Patients With Acute Respiratory Distress Syndrome. Crit Care Med. 2019 Nov;47(11):1599-1606

24. Winkler MH, Touw HR, van de Ven PM, Twisk J, Tuinman PR. Diagnostic Accuracy of Chest Radiograph, and When Concomitantly Studied Lung Ultrasound, in Critically III Patients With Respiratory Symptoms: A Systematic Review and Meta-Analysis. Crit Care Med. 2018 Jul;46(7):e707-e714.

\section{Figures}




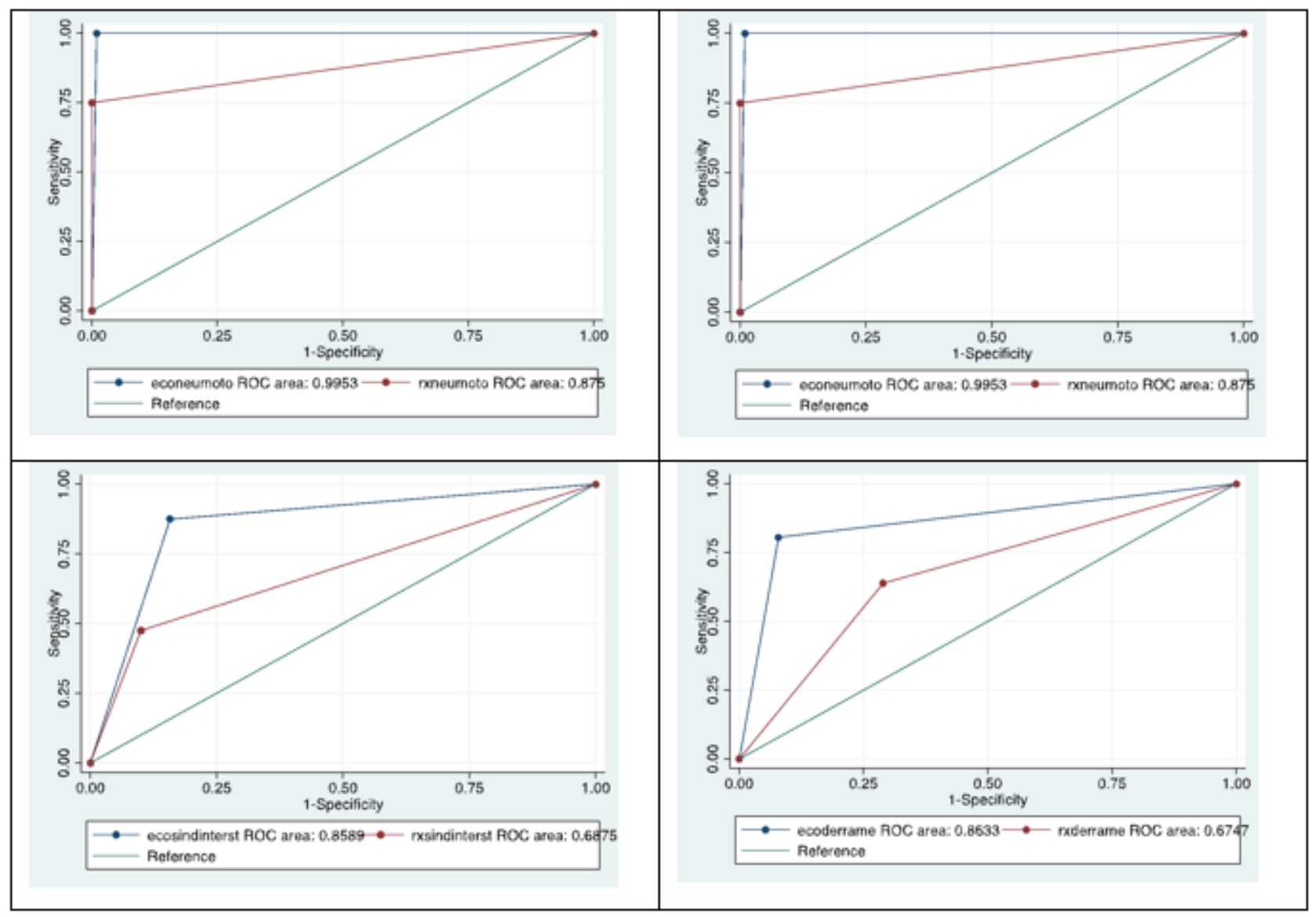

Figure 1

Sensitivity and specificity of the 